\title{
COUNTING CUSPS ON COMPLETE MANIFOLDS OF FINITE VOLUME
}

\author{
Peter Li ANd Jiaping WANG
}

\section{$\S 0$ Introduction}

In this article, we consider complete, n-dimensional, Riemannian manifolds of finite volume. We assume that the Ricci curvature is bounded from below and normalized to have the lower bound given by

$$
\operatorname{Ric}_{M} \geq-(n-1) .
$$

Since $M$ has finite volume, the constant functions are $L^{2}$ harmonic functions, implying that 0 is an eigenvalue for the $L^{2}$-spectrum of the Laplacian. We define the quantity $\mu_{1}(M)$ by the Rayleigh quotient

$$
\mu_{1}(M)=\inf _{\phi \in H^{1}(M), \int_{M}} \frac{\int_{M}|\nabla \phi|^{2}}{\int_{M} \phi^{2}},
$$

where the infimum is taken over all functions $\phi$ in the Sobolev space $H^{1}(M)$ satisfying $\int_{M} \phi=0$. This plays the role of a generalized first non-zero Neumann eigenvalue, although $\mu_{1}(M)$ might not necessarily be an eigenvalue. Note that

$$
\mu_{1}(M) \leq \max \left\{\lambda_{1}\left(\Omega_{1}\right), \lambda_{1}\left(\Omega_{2}\right)\right\},
$$

for any two disjoint domains $\Omega_{1}$ and $\Omega_{2}$ of $M$, where $\lambda_{1}\left(\Omega_{1}\right)$ and $\lambda_{1}\left(\Omega_{2}\right)$ are their first Dirichlet eigenvalues respectively. In particular, we have $\mu_{1}(M) \leq \lambda_{\text {ess }}(M)$, the smallest essential spectrum of $M$. Therefore, according to a result of Cheng [C], one always has

$$
\mu_{1}(M) \leq \frac{(n-1)^{2}}{4} .
$$

The main purpose of this paper is to prove the following theorem.

Theorem 0.1. Let $M^{n}$ be a complete Riemannian manifold with Ricci curvature bounded from below by

$$
\operatorname{Ric}_{M} \geq-(n-1) .
$$

Received by the editors March 26, 2009.

The first author was partially supported by NSF grant DMS-0801988. The second author was partially supported by NSF grant DMS-0706706. 
Assume that $M$ has finite volume given by $V$, and

$$
\mu_{1}(M) \geq \frac{(n-1)^{2}}{4} .
$$

Let us denote $N(M)$ to be the number of ends (cusps) of $M$. Then there exists a constant $C(n)>0$ depending only on $n$, such that,

$$
N(M) \leq C(n)\left(\frac{V}{V_{p}(1)}\right)^{2} \ln \left(\frac{V}{V_{p}(1)}\right),
$$

where $V_{p}(1)$ denotes the volume of the unit ball centered at any point $p \in M$.

The assumption $\mu_{1}(M) \geq \frac{(n-1)^{2}}{4}$ implies that 0 is the only eigenvalue below $\frac{(n-1)^{2}}{4}$. So the spectrum of $M$ satisfies $\sigma(M) \subset\{0\} \cup\left[\frac{(n-1)^{2}}{4}, \infty\right)$.

In the special case when $n=2$, our estimate is less effective than using the CohnVossen-Hartman formula (see [LT]). Indeed, the assumption that the sectional curvature $K \geq-1$ implies that the negative part of the curvature defined by

$$
K_{-}=\left\{\begin{array}{rll}
0 & \text { if } \quad K>0 \\
-K & \text { if } \quad K \leq 0
\end{array}\right.
$$

is at most 1. In particular,

$$
\int_{M} K_{-} \leq V(M)
$$

and $M$ has finite total curvature. Hartman's theorem then implies that $M$ must be conformally equivalent to a compact Riemann surface of genus $g$ with $N(M)$ punctures. Moreover, since $M$ has finite volume, the Cohn-Vossen-Hartman formula (see $[\mathrm{LT}])$ asserts that

$$
\begin{aligned}
-V(M) & \leq \int_{M} K \\
& =2 \pi \chi(M) .
\end{aligned}
$$

In particular, since the Euler characteristic is given by

$$
\chi(M)=2-2 g-N(M)
$$

we conclude that

$$
N(M) \leq 2-2 g+(2 \pi)^{-1} V(M) .
$$

This indicates that the dependency in $V(M)$ is better than the one provided by Theorem 0.1, and the value of the theorem lies in the cases when $n \geq 3$.

Note that if $M=\mathbb{H}^{n} / \Gamma$ is a hyperbolic manifold, with its universal covering given by the hyperbolic $n$-space $\mathbb{H}^{n}$. The $L^{2}$-spectrum of the Laplacian on $\mathbb{H}^{n}$ is the interval $\left[\frac{(n-1)^{2}}{4}, \infty\right)$. In this special case, the assumption that $\mu_{1}(M) \geq \frac{(n-1)^{2}}{4}$ can be expressed as $\mu_{1}(M)=\lambda_{1}\left(\mathbb{H}^{n}\right)$, where $\lambda_{1}\left(\mathbb{H}^{n}\right)$ is the greatest lower bound of the spectrum of $\Delta$ on $\mathbb{H}^{n}$. With this point of view, it is also possible to prove a theorem 
analogous to Theorem 0.1 for locally symmetric spaces of finite volume. Moreover, after applying Margulis lemma, one concludes that the number of ends can be estimated from above by a quantity depending on the volume of the manifold and its dimension alone. However, stronger results are available by using the thick-thin decomposition. For any finite volume locally symmetric space $M=X / \Gamma$, where $X$ is a symmetric space and $\Gamma$ a discrete subgroup of the isometry group of $X$, the number of ends of $M$ is always bounded by $N(M) \leq C(X) V$, where the constant $C$ depends on $X$ and $V$ is the volume of $M$. Indeed, in general, the total topology of $M$ is also controlled by the volume of $M$ alone (see [G]).

\section{$\S 1$ Preliminaries}

Let us first recall an estimate first proved by the authors in [LW1] and improved in [LW4]. For our purpose, it is important to know the precise dependency of the constant in the final estimate. For the sake of completeness, we will outline the proof and keep track of the dependency of all the constants. Throughout this article, we denote the quantity

$$
\lambda_{1}(E)=\inf _{\phi \in H^{1}(M),\left.\phi\right|_{\partial E}=0} \frac{\int_{E}|\nabla \phi|^{2}}{\int_{E} \phi^{2}}
$$

to be the greatest lower bound of the $L^{2}$-spectrum of the Dirichlet Laplacian on the manifold $E$.

Theorem 1.1. Let $M$ be a complete Riemannian manifold. Suppose $E$ is an end of $M$ such that $\lambda_{1}(E)>\mu$, for some constant $\mu$. Let $f$ be a nonnegative function defined on $E$ satisfying the differential inequality

$$
\Delta f \geq-\mu f
$$

If $f$ satisfies the growth condition

$$
\int_{E(R)} f^{2} e^{-2 a r}=o(R)
$$

as $R \rightarrow \infty$, with $a=\sqrt{\lambda_{1}(E)-\mu}$, then it must satisfy the decay estimate

$$
\int_{E(R+1) \backslash E(R)} f^{2} \leq C(a)\left(1+\left(R-R_{0}\right)^{-1}\right) e^{-2 a R} \int_{E\left(R_{0}+1\right) \backslash E\left(R_{0}\right)} e^{2 a r} f^{2}
$$

for some constant $C(a)>0$ depending only on a and for all $R \geq 2\left(R_{0}+1\right)$, where $E(R)=B_{p}(R) \cap E$.

Proof. We will first prove that for any $0<\delta<1$, there exists a constant $0<C<\infty$ such that,

$$
\int_{E} e^{2 \delta a r} f^{2} \leq C
$$


Indeed, let $\phi(r(x))$ be a non-negative cut-off function where support on $E$ with $r(x)$ is the geodesic distance to the fixed point $p$. Then for any function, $h(r(x))$, integration by parts yields

$$
\begin{aligned}
\int_{E} \mid \nabla & \left.\left(\phi e^{h} f\right)\right|^{2} \\
& =\int_{E}\left|\nabla\left(\phi e^{h}\right)\right|^{2} f^{2}+\int_{E}\left(\phi e^{h}\right)^{2}|\nabla f|^{2}+2 \int_{E} \phi e^{h} f\left\langle\nabla\left(\phi e^{h}\right), \nabla f\right\rangle \\
& =\int_{E}\left|\nabla\left(\phi e^{h}\right)\right|^{2} f^{2}+\int_{E} \phi^{2} e^{2 h}|\nabla f|^{2}+\frac{1}{2} \int_{E}\left\langle\nabla\left(\phi^{2} e^{2 h}\right), \nabla\left(f^{2}\right)\right\rangle \\
= & \int_{E}\left|\nabla\left(\phi e^{h}\right)\right|^{2} f^{2}+\int_{E} \phi^{2} e^{2 h}|\nabla f|^{2}-\frac{1}{2} \int_{E} \phi^{2} e^{2 h} \Delta\left(f^{2}\right) \\
\leq & \int_{E}\left|\nabla\left(\phi e^{h}\right)\right|^{2} f^{2}+\mu \int_{E} \phi^{2} e^{2 h} f^{2} \\
= & \int_{E}|\nabla \phi|^{2} e^{2 h} f^{2}+2 \int_{E} \phi e^{2 h}\langle\nabla \phi, \nabla h\rangle f^{2} \\
& \quad+\int_{E} \phi^{2}|\nabla h|^{2} e^{2 h} f^{2}+\mu \int_{E} \phi^{2} e^{2 h} f^{2} .
\end{aligned}
$$

On the other hand, using the variational principle for $\lambda_{1}(E)$, we have

$$
\lambda_{1}(E) \int_{E} \phi^{2} e^{2 h} f^{2} \leq \int_{E}\left|\nabla\left(\phi e^{h} f\right)\right|^{2},
$$

hence (1.1) becomes

$$
\begin{aligned}
& a^{2} \int_{E} \phi^{2} e^{2 h} f^{2} \\
& \quad \leq \int_{E}|\nabla \phi|^{2} e^{2 h} f^{2}+2 \int_{E} \phi e^{2 h}\langle\nabla \phi, \nabla h\rangle f^{2}+\int_{E} \phi^{2}|\nabla h|^{2} e^{2 h} f^{2} .
\end{aligned}
$$

Let us now choose

$$
\phi(r(x))=\left\{\begin{array}{ccc}
r(x)-R_{0} & \text { on } & E\left(R_{0}+1\right) \backslash E\left(R_{0}\right) \\
1 & \text { on } & E(R) \backslash E\left(R_{0}+1\right), \\
R^{-1}(2 R-r(x)) & \text { on } & E(2 R) \backslash E(R) \\
0 & \text { on } & E \backslash E(2 R),
\end{array}\right.
$$

and

$$
h(r)=\left\{\begin{array}{lrrr}
\delta a r & \text { for } & r \leq \frac{K}{(1+\delta) a} \\
K-a r & \text { for } & r \geq \frac{K}{(1+\delta) a}
\end{array}\right.
$$


for some fixed $K>\left(R_{0}+1\right)(1+\delta) a$. When $R \geq \frac{K}{(1+\delta) a}$, we see that

$$
|\nabla h|^{2}=\left\{\begin{array}{llrl}
\delta^{2} a^{2} & \text { for } & & r \leq \frac{K}{(1+\delta) a} \\
a^{2} & \text { for } & r \geq \frac{K}{(1+\delta) a}
\end{array}\right.
$$

and

$$
\langle\nabla \phi, \nabla h\rangle=\left\{\begin{array}{ccc}
\delta a & \text { on } \quad E\left(R_{0}+1\right) \backslash E\left(R_{0}\right) \\
R^{-1} a & \text { on } \quad E(2 R) \backslash E(R) \\
0 & \text { otherwise. }
\end{array}\right.
$$

Substituting into (1.2), we obtain

$$
\begin{aligned}
& a^{2} \int_{E} \phi^{2} e^{2 h} f^{2} \\
& \leq \int_{E\left(R_{0}+1\right) \backslash E\left(R_{0}\right)} e^{2 h} f^{2}+R^{-2} \int_{E(2 R) \backslash E(R)} e^{2 h} f^{2} \\
& \quad+2 \delta a \int_{E\left(R_{0}+1\right) \backslash E\left(R_{0}\right)} e^{2 h} f^{2}+2 R^{-1} a \int_{E(2 R) \backslash E(R)} e^{2 h} f^{2} \\
& \quad+\delta^{2} a^{2} \int_{\left.E\left(K((1+\delta) a)^{-1}\right) \backslash E\left(R_{0}\right)\right)} \phi^{2} e^{2 h} f^{2}+a^{2} \int_{E(2 R) \backslash E\left(K((1+\delta) a)^{-1}\right)} \phi^{2} e^{2 h} f^{2} .
\end{aligned}
$$

This can be rewritten as

$$
\begin{aligned}
& a^{2} \int_{E\left(K((1+\delta) a)^{-1}\right) \backslash E\left(R_{0}+1\right)} e^{2 h} f^{2} \\
& \leq a^{2} \int_{E\left(K((1+\delta) a)^{-1}\right)} \phi^{2} e^{2 h} f^{2} \\
& \leq \int_{E\left(R_{0}+1\right) \backslash E\left(R_{0}\right)} e^{2 h} f^{2}+R^{-2} \int_{E(2 R) \backslash E(R)} e^{2 h} f^{2} \\
& \quad+2 \delta a \int_{E\left(R_{0}+1\right) \backslash E\left(R_{0}\right)} e^{2 h} f^{2}+R^{-1} \delta a \int_{E(2 R) \backslash E(R)} e^{2 h} f^{2} \\
& \quad+\delta^{2} a^{2} \int_{\left.E\left(K((1+\delta) a)^{-1}\right) \backslash E\left(R_{0}\right)\right)} \phi^{2} e^{2 h} f^{2},
\end{aligned}
$$

hence

$$
\begin{aligned}
& \left(1-\delta^{2}\right) a^{2} \int_{\left.E\left(K((1+\delta) a)^{-1}\right) \backslash E\left(R_{0}+1\right)\right)} e^{2 h} f^{2} \\
& \leq\left(\delta^{2} a^{2}+2 \delta a+1\right) \int_{\left.E\left(R_{0}+1\right) \backslash E\left(R_{0}\right)\right)} e^{2 h} f^{2} \\
& \quad+R^{-2} \int_{E(2 R) \backslash E(R)} e^{2 h} f^{2}+2 R^{-1} a \int_{E(2 R) \backslash E(R)} e^{2 h} f^{2} .
\end{aligned}
$$


The definition of $h$ and the assumption on the growth estimate on $f$ imply that the last two terms on the right hand side tends to 0 as $R \rightarrow \infty$. Hence we obtain the estimate

$$
\left(1-\delta^{2}\right) a^{2} \int_{\left.E\left(K((1+\delta) a)^{-1}\right) \backslash E\left(R_{0}+1\right)\right)} e^{2 \delta a r} f^{2} \leq\left(\delta^{2} a^{2}+2 \delta a+1\right) \int_{\left.E\left(R_{0}+1\right) \backslash E\left(R_{0}\right)\right)} e^{2 \delta a r} f^{2} .
$$

Since the right hand side is independent of $K$, by letting $K \rightarrow \infty$ we conclude that

$$
\int_{E \backslash E\left(R_{0}+1\right)} e^{2 \delta a r} f^{2} \leq C_{1},
$$

with

$$
C_{1}=\frac{\left(\delta^{2} a^{2}+2 \delta a+1\right)}{\left(1-\delta^{2}\right) a^{2}} \int_{\left.E\left(R_{0}+1\right) \backslash E\left(R_{0}\right)\right)} e^{2 \delta a r} f^{2} .
$$

Our next step is to improve this estimate by setting $h=a r$ in the preceding argument. Note that (1.2) asserts that

$$
\begin{aligned}
-2 a & \int_{E} \phi e^{2 a r}\langle\nabla \phi, \nabla r\rangle f^{2} \\
& \leq \int_{E}|\nabla \phi|^{2} e^{2 a r} f^{2} .
\end{aligned}
$$

For $R_{0}<R_{1}<R$, let us choose $\phi$ to be

$$
\phi(x)=\left\{\begin{array}{lll}
\frac{r(x)-R_{0}}{R_{1}-R_{0}} & \text { on } & E\left(R_{1}\right) \backslash E\left(R_{0}\right) \\
\frac{R-r(x)}{R-R_{1}} & \text { on } & E(R) \backslash E\left(R_{1}\right) .
\end{array}\right.
$$

We conclude that

$$
\begin{aligned}
\frac{2 a}{\left(R-R_{1}\right)^{2}} & \int_{E(R) \backslash E\left(R_{1}\right)}(R-r(x)) e^{2 a r} f^{2} \\
\leq & \frac{1}{\left(R_{1}-R_{0}\right)^{2}} \int_{E\left(R_{1}\right) \backslash E\left(R_{0}\right)} e^{2 a r} f^{2}+\frac{1}{\left(R-R_{1}\right)^{2}} \int_{E(R) \backslash E\left(R_{1}\right)} e^{2 a r} f^{2} \\
& \quad+\frac{2 a}{\left(R_{1}-R_{0}\right)^{2}} \int_{E\left(R_{1}\right) \backslash E\left(R_{0}\right)}\left(r-R_{0}\right) e^{2 a r} f^{2} .
\end{aligned}
$$

On the other hand, for any $0<t<R-R_{1}$, since

$$
\begin{aligned}
& \frac{2 a t}{\left(R-R_{1}\right)^{2}} \int_{E(R-t) \backslash E\left(R_{1}\right)} e^{2 a r} f^{2} \\
& \quad \leq \frac{2 a}{\left(R-R_{1}\right)^{2}} \int_{E(R) \backslash E\left(R_{1}\right)}(R-r(x)) e^{2 a r} f^{2},
\end{aligned}
$$


we deduce that

$$
\begin{aligned}
& \frac{2 a t}{\left(R-R_{1}\right)^{2}} \int_{E(R-t) \backslash E\left(R_{1}\right)} e^{2 a r} f^{2} \\
& \leq\left(\frac{2 a}{R_{1}-R_{0}}+\frac{1}{\left(R_{1}-R_{0}\right)^{2}}\right) \int_{E\left(R_{1}\right) \backslash E\left(R_{0}\right)} e^{2 a r} f^{2} \\
& +\frac{1}{\left(R-R_{1}\right)^{2}} \int_{E(R) \backslash E\left(R_{1}\right)} e^{2 a r} f^{2} .
\end{aligned}
$$

Observe that if we take $R_{1}=R_{0}+1, t=a^{-1}$, and set

$$
g(R)=\int_{E(R) \backslash E\left(R_{0}+1\right)} e^{2 a r} f^{2},
$$

then inequality (1.4) can be written as

$$
g\left(R-a^{-1}\right) \leq C_{2} R^{2}+\frac{1}{2} g(R)
$$

where

$$
C_{2}=\frac{2 a+1}{2} \int_{E\left(R_{0}+1\right) \backslash E\left(R_{0}\right)} e^{2 a r} f^{2}
$$

is independent of $R$. Iterating this inequality, we obtain for any positive integer $k$ and $R \geq 1$

$$
\begin{aligned}
g(R) & \leq C_{2} \sum_{i=1}^{k} \frac{\left(R+i a^{-1}\right)^{2}}{2^{i-1}}+2^{-k} g\left(R+k a^{-1}\right) \\
& \leq C_{2} R^{2} \sum_{i=1}^{\infty} \frac{\left(1+i a^{-1}\right)^{2}}{2^{i-1}}+2^{-k} g\left(R+k a^{-1}\right) \\
& \leq C_{3} R^{2}+2^{-k} g\left(R+k a^{-1}\right),
\end{aligned}
$$

where

$$
C_{3}=C_{2} \sum_{i=1}^{\infty} \frac{\left(1+i a^{-1}\right)^{2}}{2^{i-1}}
$$

However, our previous estimate (1.3)

$$
\int_{E} e^{2 \delta a r} f^{2} \leq C_{1}
$$

implies that

$$
\begin{aligned}
g\left(R+k a^{-1}\right) & =\int_{E\left(R+k a^{-1}\right) \backslash E\left(R_{0}+1\right)} e^{2 a r} f^{2} \\
& \leq e^{2\left(R+k a^{-1}\right)(1-\delta)} \int_{E\left(R+k a^{-1}\right) \backslash E\left(R_{0}+1\right)} e^{2 \delta a r} f^{2} \\
& \leq C_{1} e^{2\left(R+k a^{-1}\right)(1-\delta)} .
\end{aligned}
$$


Hence,

$$
2^{-k} g\left(R+k a^{-1}\right) \rightarrow 0
$$

as $k \rightarrow \infty$ by choosing $2(1-\delta) a^{-1}<\ln 2$. This proves the estimate

$$
\int_{E(R) \backslash E\left(R_{0}+1\right)} e^{2 a r} f^{2} \leq C_{3} R^{2}
$$

Using inequality (1.4) again and by choosing $R_{1}=R_{0}+1$ and $t=\frac{R}{2}$ this time, we conclude that

$$
\begin{aligned}
& a R \int_{E\left(\frac{R}{2}\right) \backslash E\left(R_{0}+1\right)} e^{2 a r} f^{2} \\
& \quad \leq(2 a+1)\left(R-R_{0}-1\right)^{2} \int_{E\left(R_{0}+1\right) \backslash E\left(R_{0}\right)} e^{2 a r} f^{2}+\int_{E(R) \backslash E\left(R_{0}+1\right)} e^{2 a r} f^{2} .
\end{aligned}
$$

Applying the estimate (1.5) to the second term on the right hand side, we have

$$
\int_{E\left(\frac{R}{2}\right) \backslash E\left(R_{0}+1\right)} e^{2 a r} f^{2} \leq C_{4} R
$$

where

$$
C_{4}=(2 a+1) a^{-1}\left(\frac{\left(R-R_{0}-1\right)^{2}}{R^{2}}+\sum_{i=1}^{\infty} \frac{\left(1+i a^{-1}\right)^{2}}{2^{i}}\right) \int_{E\left(R_{0}+1\right) \backslash E\left(R_{0}\right)} e^{2 a r} f^{2} .
$$

Therefore, for $R \geq 2\left(R_{0}+1\right)$,

$$
\int_{E(R)} e^{2 a r} f^{2} \leq C_{5} R
$$

with

$$
C_{5}=C(a) \int_{E\left(R_{0}+1\right) \backslash E\left(R_{0}\right)} e^{2 a r} f^{2},
$$

where $C(a)$ denotes a constant depending only on $a$. We are now ready to prove the lemma by using (1.6). Setting $t=2 a^{-1}$ and $R_{1}=R-4$ in (1.4), we obtain

$$
\begin{aligned}
& \int_{E(R-2) \backslash E(R-4)} e^{2 a r} f^{2} \\
& \leq\left(\frac{8 a}{R-R_{0}-4}+\frac{4}{\left(R-R_{0}-4\right)^{2}}\right) \int_{E(R-4) \backslash E\left(R_{0}\right)} e^{2 a r} f^{2} \\
& \quad+\frac{1}{4} \int_{E(R) \backslash E(R-4)} e^{2 a r} f^{2} .
\end{aligned}
$$


According to (1.6), the first term of the right hand side is bounded by

$$
C_{5}\left(C(a)+\left(R-R_{0}-4\right)^{-1}\right)
$$

for $R-4 \geq 2\left(R_{0}+1\right)$. Hence, by renaming $R$, the above inequality can be rewritten as

$$
\int_{E(R-2) \backslash E(R-4)} e^{2 a r} f^{2} \leq C_{5}\left(C(a)+\left(R-R_{0}\right)^{-1}\right)+\frac{1}{3} \int_{E(R+4) \backslash E(R+2)} e^{2 a r} f^{2} .
$$

Iterating this inequality $k$ times, we arrive at

$$
\begin{aligned}
& \int_{E(R+2) \backslash E(R)} e^{2 a r} f^{2} \\
& \quad \leq C_{5}\left(C(a)+\left(R-R_{0}\right)^{-1}\right) \sum_{i=0}^{k-1} 3^{-i}+3^{-k} \int_{E(R+2(k+1)) \backslash E(R+2 k)} e^{2 a r} f^{2} .
\end{aligned}
$$

However, using (1.6) again, we conclude that the second term is bounded by

$$
3^{-k} \int_{E(R+2(k+1)) \backslash E(R+2 k)} e^{2 a r} f^{2} \leq C_{5} 3^{-k}(R+2(k+1))
$$

which tends to 0 as $k \rightarrow \infty$. Hence

$$
\int_{E(R+2) \backslash E(R)} e^{2 a r} f^{2} \leq C_{5}\left(C(a)+\left(R-R_{0}\right)^{-1}\right)
$$

for $r \geq 2\left(R_{0}+1\right)$, and the theorem follows.

Recall that an end $E$ is said to be parabolic if it does not admit any positive Green's function satisfying the Neumann boundary conditions on its boundary $\partial E$. In [LW1], it was pointed out that in the case $\lambda_{1}(E)>0$, the end $E$ is parabolic if and only if its volume is finite.

Corollary 1.2. Let $E$ be an end of complete manifold $M$ with $\lambda_{1}(E)>0$. If $E$ is a parabolic end, then $E$ must have exponential volume decay given by

$$
\begin{aligned}
& V_{E}(R+1)-V_{E}(R) \\
\leq & C\left(\lambda_{1}(E)\right)\left(1+\left(R-R_{0}\right)^{-1}\right) \exp \left(2 \sqrt{\lambda_{1}(E)}\left(R_{0}-R\right)\right)\left(V_{E}\left(R_{0}+1\right)-V_{E}\left(R_{0}\right)\right)
\end{aligned}
$$

for some constant $C\left(\lambda_{1}(E)\right)>0$ depending on $\lambda_{1}(E)$.

Proof. The corollary follows from Theorem 1.1 by setting the function $f$ to be the constant function 1.

Note that the corollary can be applied in particular to the case $E=M \backslash B_{p}\left(R_{0}\right)$, where $B_{p}\left(R_{0}\right)$ is the geodesic ball of radius $R_{0}$ centered at a fixed point $p$ in $M$. This will give a volume decay estimate for the annulus $B_{p}(R+1) \backslash B_{p}(R)$ for all $R>R_{0}$. 


\section{$\S 2$ Proof of Theorem 0.1}

We now apply the estimate from the previous section to prove the following result.

Theorem 2.1. Let $M^{n}$ be a complete Riemannian manifold with Ricci curvature bounded from below by

$$
\operatorname{Ric}_{M} \geq-(n-1) .
$$

Assume that $M$ has finite volume given by $V$, and

$$
\lambda_{1}\left(M \backslash B_{p}\left(R_{0}\right)\right) \geq \frac{(n-1)^{2}}{4} .
$$

Then there exists a constant $C(n)>0$ depending only on $n$, such that, the number of ends of $M$ satisfies

$$
N(M) \leq C(n) V V_{p}^{-1}(1) \exp \left((n-1) R_{0}\right),
$$

where $V_{p}(1)$ denotes the volume of the unit ball centered at a point $p \in M$.

Proof. According to Corollary 1.2, if we denote $V_{p}(R)$ to be the volume of the geodesic ball $B_{p}(R)$, then for all $R \geq 2\left(R_{0}+1\right)$ we have

$V_{p}(R+2)-V_{p}(R) \leq C\left(1+\left(R-R_{0}\right)^{-1}\right) \exp \left((n-1)\left(R_{0}-R\right)\right)\left(V_{p}\left(R_{0}+1\right)-V_{p}\left(R_{0}\right)\right)$.

On the other hand, the standard volume comparison asserts that if $y \in \partial B_{p}(R+1)$ then

$$
V_{y}(1) \geq C_{1}^{-1} \exp (-(n-1) R) V_{p}(1)
$$

Obviously, if $N(R)$ denotes the number of ends with respect to $B_{p}(R)$, namely $M \backslash$ $B_{p}(R)$ has $N(R)$ unbounded components, then there exist $N(R)$ number of points $\left\{y_{i} \in \partial B_{p}(R)\right\}$ such that $B_{y_{i}}(1) \cap B_{y_{j}}(1)=\emptyset$ for $i \neq j$. In particular applying (2.2) to each of the $y_{i}$ and combining with (2.1), we conclude that

$$
\begin{aligned}
N(R) C_{1}^{-1} & \exp (-(n-1) R) V_{p}(1) \\
& \leq \sum_{i=1}^{N(R)} V_{y_{i}}(1) \\
& \leq V_{p}(R+2)-V_{p}(R) \\
& \leq C\left(1+\left(R-R_{0}\right)^{-1}\right) \exp \left((n-1)\left(R_{0}-R\right)\right)\left(V_{p}\left(R_{0}+1\right)-V_{p}\left(R_{0}\right)\right) .
\end{aligned}
$$

This implies that

$$
N(R) \leq C C_{1}\left(1+\left(R-R_{0}\right)^{-1}\right) \exp \left((n-1) R_{0}\right)\left(V_{p}\left(R_{0}+1\right)-V_{p}\left(R_{0}\right)\right) V_{p}^{-1}(1) .
$$

Letting $R \rightarrow \infty$, we conclude that the number of ends $N(M)$ of $M$ is bounded by

$$
N(M) \leq C C_{1} \exp \left((n-1) R_{0}\right)\left(V_{p}\left(R_{0}+1\right)-V_{p}\left(R_{0}\right)\right) V_{p}^{-1}(1),
$$


and the result follows.

We remark that if $M$ has finitely many eigenvalues $0=\lambda_{1}<\lambda_{2} \leq \cdots \leq \lambda_{k}$ below $\frac{(n-1)^{2}}{4}$, then it is easy to see there exists $R_{0}>0$ such that

$$
\lambda_{1}\left(M \backslash B_{p}\left(R_{0}\right)\right) \geq \frac{(n-1)^{2}}{4} .
$$

In particular, Theorem 2.1 implies that $M$ must have finitely many ends. However, the estimate on the number of ends is not effective as it is unclear to us at this moment how to control the size of $R_{0}$ in terms of the eigenvalues below $\frac{(n-1)^{2}}{4}$. On the other hand, we will demonstrate below that $R_{0}$ can be effectively controlled if $k=1$.

We also remark that the proof of Theorem 2.1 can be applied to any finite volume locally symmetric space $M=X / \Gamma$. Indeed, it is known in this case that there exists $R_{0}>0$ such that $\lambda_{1}\left(M \backslash B_{p}\left(R_{0}\right)\right)=\lambda_{1}(X)$ and an appropriate version of the volume comparison theorem (in terms of the balls defined by the Busemann functions) holds on $M$. So such $M$ must have finitely many ends. Of course, as pointed out in the introduction, stronger result holds true. In passing, we mention for such $M$, whether $\mu_{1}(M)=\lambda_{1}(X)$ is an important question. The famous Selberg conjecture says this is the case if $M$ is an arithmetic surface.

The following lemma allows us to estimate $\lambda_{1}\left(B_{p}(R)\right)$ of a geodesic ball centered at $p$ with radius $R$ in terms of the volume of the ball. Note that we do not need to impose any curvature assumptions on $M$.

Lemma 2.2. Let $M$ be a complete Riemannian manifold. Then for any $0<\delta<1$, $R>2$ and $p \in M$, we have

$$
\lambda_{1}\left(B_{p}(R)\right) \leq \frac{1}{4 \delta^{2}(R-1)^{2}}\left(\ln \left(\frac{V_{p}(R)}{V_{p}(1)}\right)+\ln \left(\frac{81}{1-\delta}\right)\right)^{2} .
$$

Proof. We use $\lambda_{1}$ to denote $\lambda_{1}\left(B_{p}(R)\right)$ to ease the notations. We may assume

$$
\lambda_{1} \geq \frac{4}{R^{2}}
$$

as otherwise the conclusion automatically holds true. Let $r(x)$ denote the distance function to a fixed point $p \in M$. The variational characteristic of $\lambda_{1}\left(B_{p}(R)\right)$ implies that

$$
\begin{aligned}
\lambda_{1}\left(B_{p}(R)\right) \int_{M} \phi^{2} \exp \left(-2 \delta \sqrt{\lambda_{1}} r\right) \\
\leq \int_{M} \mid \nabla\left(\left.\phi \exp \left(-\delta \sqrt{\lambda_{1}} r\right)\right|^{2}\right. \\
=\int_{M}|\nabla \phi|^{2} \exp \left(-2 \delta \sqrt{\lambda_{1}} r\right)-2 \delta \sqrt{\lambda_{1}} \int_{M} \phi \exp \left(-2 \delta \sqrt{\lambda_{1}} r\right)\langle\nabla \phi, \nabla r\rangle \\
\quad+\delta^{2} \lambda_{1} \int_{M} \phi^{2} \exp \left(-2 \delta \sqrt{\lambda_{1}} r\right)
\end{aligned}
$$


for any nonnegative Lipschitz function $\phi$ with support in $B_{p}(R)$. In particular, for $R>2$, if we choose

$$
\phi=\left\{\begin{array}{ccl}
1 & \text { on } & B_{p}\left(R-\lambda_{1}^{-\frac{1}{2}}\right) \\
\sqrt{\lambda_{1}}(R-r) & \text { on } & B_{p}(R) \backslash B_{p}\left(R-\lambda_{1}^{-\frac{1}{2}}\right), \\
0 & \text { on } & M \backslash B_{p}(R)
\end{array}\right.
$$

then we have

$$
\begin{aligned}
\left(1-\delta^{2}\right) \lambda_{1} & \exp \left(-2 \delta \sqrt{\lambda_{1}}\right) V_{p}(1) \\
& \leq\left(1-\delta^{2}\right) \lambda_{1} \int_{M} \phi^{2} \exp \left(-2 \delta \sqrt{\lambda_{1}} r\right) \\
& =\int_{M}|\nabla \phi|^{2} \exp \left(-2 \delta \sqrt{\lambda_{1}} r\right)-2 \delta \sqrt{\lambda_{1}} \int_{M} \phi \exp \left(-2 \delta \sqrt{\lambda_{1}} r\right)\langle\nabla \phi, \nabla r\rangle \\
& \leq(1+2 \delta) \lambda_{1} \exp \left(-2 \delta\left(\sqrt{\lambda_{1}} R-1\right)\right) V_{p}(R) .
\end{aligned}
$$

Therefore,

$$
\exp \left(2 \delta\left(\sqrt{\lambda_{1}}(R-1)\right)\right) \leq \frac{27}{1-\delta} \frac{V_{p}(R)}{V_{p}(1)}
$$

and

$$
2 \delta \sqrt{\lambda_{1}}(R-1) \leq \ln \left(\frac{27}{1-\delta}\right)+\ln \left(\frac{V_{p}(R)}{V_{p}(1)}\right) .
$$

The lemma follows by rewriting this inequality.

As a corollary of this lemma, the bottom spectrum of any complete manifold $M$ can be estimated in terms of its volume entropy. This result was proved in [LW1] with a different argument.

Corollary 2.3. Let $M$ be a complete manifold and $\lambda_{1}(M)$ its bottom spectrum. Then

$$
\lambda_{1}(M) \leq \frac{1}{4}\left(\liminf _{R \rightarrow \infty} \frac{\ln V_{p}(R)}{R}\right)^{2} .
$$

Proof. Note that $\lambda_{1}(M)=\lim _{R \rightarrow \infty} \lambda_{1}\left(B_{p}(R)\right)$. Now the result follows by first letting $R$ go to infinity and then $\delta$ go to 1 in the estimate of Lemma 2.2 .

We now prove Theorem 0.1 .

Proof of Theorem 0.1. Let $p \in M$ be a fixed point. For any $0<\delta<1$, let

$$
R_{0}=\frac{1}{(n-1) \delta}\left(\ln \left(\frac{81}{1-\delta}\right)+\ln \left(\frac{V}{V_{p}(1)}\right)\right)+3 .
$$

Then according to Lemma 2.2, 


$$
\lambda_{1}\left(B_{p}\left(R_{0}\right)\right) \leq \frac{(n-1)^{2}}{4}
$$

We now observe that

$$
\lambda_{1}\left(M \backslash B_{p}\left(R_{0}\right)\right) \geq \frac{(n-1)^{2}}{4} .
$$

Indeed, if (2.4) is valid and also $\lambda_{1}\left(M \backslash B_{p}\left(R_{0}\right)\right)<\frac{(n-1)^{2}}{4}$, then the variational principle will imply that $\left.\mu_{1}(M)<\frac{(n-1)^{2}}{4}\right)$, contradicting our assumption.

By Theorem 2.1, we have

$$
N(M) \leq C(n) V V_{p}^{-1}(1) \exp \left((n-1) R_{0}\right) .
$$

The claimed estimate then follows by plugging in the value of $R_{0}$ and setting

$$
\delta=1-\frac{1}{\ln \left(V V_{p}^{-1}(1)\right)} .
$$

As pointed out earlier, the key ingredients in the proof of Theorem 0.1 relied on the decay estimate of the volume (2.2) given by the upper bound of the greatest lower bound of the spectrum of the model manifold hyperbolic space $\mathbb{H}^{n}$. The following two theorems follow similarly by using the corresponding comparison results from [LW3] and [KLZ], respectively.

Theorem 2.4. Let $M^{m}$ be a complete Kähler manifold of complex dimension $m$. Assume that $M$ has finite volume and its holomorphic bisectional curvature satisfies the bound

$$
R_{i \bar{j} \bar{i} \bar{j}} \geq-\left(1+\delta_{i j}\right)
$$

for all unitary frame $\left\{e_{i}, \ldots, d_{m}\right\}$. Suppose that $\mu_{1}(M) \geq m^{2}$, then there exists a constant $C(m)>0$ depending only on $m$, such that, the number of ends of $M$ is bounded above by

$$
N(M) \leq C(m)\left(\frac{V}{V_{p}(1)}\right)^{2} \ln \left(\frac{V}{V_{p}(1)}\right) .
$$

Theorem 2.5. Let $M^{m}$ be a complete quarternionic Kähler manifold of real dimension $4 m$. Assume that $M$ has finite volume and its scalar curvature of satisfies the bound

$$
S_{M} \geq-16 m(m+2),
$$

Suppose that $\mu_{1}(M) \geq(2 m+1)^{2}$, then there exists a constant $C(m)>0$ depending only on $m$, such that, the number of ends of $M$ is bounded above by

$$
N(M) \leq C(m)\left(\frac{V}{V_{p}(1)}\right)^{2} \ln \left(\frac{V}{V_{p}(1)}\right) .
$$


We would like to point out that in Theorem 2.4, it is reasonable to ask if the assumption on the holomorphic bisectional curvarture can be replace with the weaker assumption that the Ricci curvature is bounded from below by

$$
\operatorname{Ric}_{M} \geq-2(m+1)
$$

A theorem of Munteanu $[\mathrm{M}]$ asserted that $\lambda_{1}(M) \leq m^{2}$. However, in this case, the required volume estimate of the form

$$
V_{y}(1) \geq C_{1}^{-1} \exp (-2 m R) V_{p}(1)
$$

is not known since a volume comparison theorem has not been proven with the weaker curvature assumption.

\section{References}

[C] S. Y. Cheng, Eigenvalue comparison theorems and its geometric applications, Math. Z. 143 (1975), 289-297.

[G] T. Gelander, Homotopy type and volume of locally symmetric manifolds, Duke Math. J. 124 (2004), 459-515.

[KLZ] S. Kong, P. Li and D. Zhou, Spectrum of the Laplacian on quaternionic Kähler manifolds, J. Diff. Geom. 78 (2008), 295-332.

[LT] P. Li and L. F. Tam, Complete surfaces with finite total curvature, J. Diff. Geom. 33 (1991), 139-168.

[LW1] P. Li and J. Wang, Complete manifolds with positive spectrum, J. Diff. Geom. 58 (2001), 501-534.

[LW2] P. Li and J. Wang, Complete manifolds with positive spectrum, II., J. Diff. Geom. 62 (2002), $143-162$.

[LW3] P. Li and J. Wang, Comparison theorem for Kähler manifolds and positivity of spectrum, J. Diff. Geom. 69 (2005), 43-74.

[LW4] P. Li and J. Wang, Weighted Poincaré inequality and rigidity of complete manifolds, Ann. Scient. Éc. Norm. Sup. $4^{e}$ série 39 (2006), 921-982.

[M] O. Munteanu, A sharp estimate for the bottom of the spectrum of the Laplacian on Kähler manifolds, J. Diff. Geom. 83 (2009), 163-187.

Department of Mathematics, University of California, Irvine, CA 92697-3875

E-mail address: pli@math.uci.edu

School of Mathematics, University of Minnesota, Minneapolis, MN 55455

E-mail address: jiaping@math.umn.edu 